\title{
LOS PATRONES LEXICALES VERBALES EN LOS EVENTOS DE MOVIMIENTO EN LENGUA YAQUI
}

\author{
Denisse Fiordalizo Martínez Martínez \\ Universidad de Sonora (México) \\ denissef.martinez@gmail.com \\ Zarina Estrada Fernández \\ Universidad de Sonora / Consejo Nacional de Ciencia y Tecnología - Conacyt (México) \\ zarinaef@gmail.com
}

Recibido: 26/01/2020 - Aprobado: 13/05/2021 - Publicado: 30/07/2021

DOI: doi.org/10.17533/udea.lyl.n80a16

Resumen: En este trabajo mostramos que en lengua yaqui se distinguen cuatro patrones lexicales: i) movimiento y trayectoria; ii) movimiento y manera; iii) movimiento, trayectoria y causa; y iv) movimiento, trayectoria y figura. Asimismo, determinamos que la ocurrencia de un patrón lexical tiene implicaciones en la estructura sintáctica del evento de movimiento, pues los eventos con patrones lexicales i), ii) y iv) tienen una estructura sintáctica intransitiva, donde la figura corresponde con el sujeto de la oración. Mientras que los eventos con patrón lexical iii) tienen una estructura sintáctica transitiva, donde la figura corresponde con el objeto directo.

Palabras clave: lengua yaqui; verbos de movimiento; eventos de movimiento; patrones lexicales.

\section{VERBAL LEXICAL PATTERNS IN THE EXPRESSION OF MOTION EVENTS IN YAQUI LANGUAGE}

Abstract: In this work, we show that in Yaqui language it is possible to distinguish four lexical patterns: i) motion and path; ii) motion and manner; iii) motion, path and cause; and iv) motion, path and figure. Likewise, we determine that the occurrence of lexical patterns has implications in the syntactic structure of movement events, because the events with lexical patterns i), ii), and iv) have intransitive syntactic structures, where the figure corresponds to the subject of the sentence. Meanwhile, the events with a lexical pattern iii) have transitive syntactic structures, where the figure corresponds to the direct object.

Key words: Yaqui language; motion verbs; motion events; lexical patterns.

Editores 


\section{Introducción}

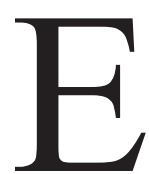

n el análisis aquí planteado se estudia la composición semántica de los verbos que ocurren en los eventos de movimiento de la lengua yaqui del estado de Sonora, México. El objetivo principal de esta investigación es determinar los patrones lexicales que muestran los verbos en la expresión de eventos de movimiento en la lengua, así como las implicaciones sintáctico-semánticas que estos tienen en el evento.

Para lograr lo anterior, se analizaron datos de cuatro fuentes: i) Jeka aniata jiapsi: jiak jamutta etejoi «Vida de Jeka Ania: historia de una mujer yaqui», obra conformada por 493 construcciones (Estrada et al., 2018); ii) Diccionario yaqui-español: repertorio de preservación lingüística, constituido por 2410 entradas léxicas (Estrada et al., 2004); iii) narraciones tradicionales yaquis, que constan de 379 construcciones (Hernández, 2002); y iv) 222 construcciones obtenidas a través de la aplicación de un cuestionario de eventos de movimiento que se elaboró a partir de las entradas verbales de movimiento identificadas en el diccionario. Dichos datos se recolectaron en un trabajo de campo realizado entre el año 2018 y 2019 con dos hablantes nativas.

\subsection{La etnia yaqui}

La etnia yaqui es una de las ocho regiones indígenas que existen en el estado de Sonora. Según el censo comunitario citado en Lerma (2014), el número total de yaquis sonorenses es de 25000 miembros. Los yaquis se autodenominan yoeme que significa «gente».

En la actualidad, la comunidad tiene presencia tanto en México como en los Estados Unidos. En México, los yaquis habitan el sur de la capital del estado de Sonora, en las inmediaciones de la sierra sonorense del Bacatete «carrizo largo». Los poblados históricos donde se localiza este grupo son: Cócorit (ko okoi «chiltepín silvestre»), Loma de Bácum (bajkom «laguna»), Tórim (tori «rata de campo»), Vícam Pueblo (bikam «puntas de flecha»), Pótam (pota «topo»), Ráhum (raajum «ebullición»), Huíribis (wiibis «pájaro huitlacoche») y Belem (beene «cuesta bajo»). Asimismo, algunos yaquis migraron a Hermosillo, la capital del estado sonorense, en la época de la Revolución mexicana y actualmente habitan en la colonia Coloso bajo (Bejípone, 2013; Moctezuma, 2007).

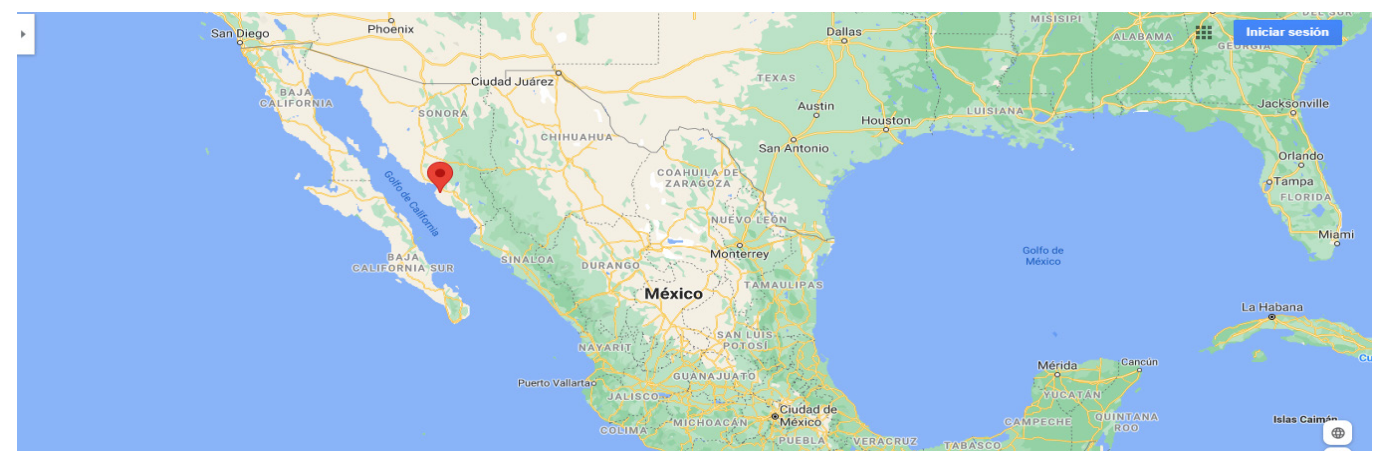

Mapa 1. Ubicación geográfica de la comunidad yaqui. Fuente: Google Maps. 


\subsection{Aspectos gramaticales de la lengua}

Autores como Lindenfeld (1973) y Dedick y Casad (1999), han descrito tipológicamente al yaqui como una lengua de estricto orden SOV, que muestra morfología aglutinante, la cual preferentemente se sufija a nombres y verbos. Respecto al alineamiento morfosintáctico, la lengua tiene un paradigma pronominal bien establecido, el cual permite distinguir un sistema nominativo-acusativo (1a). No obstante, con nombres plenos la lengua diferencia el alineamiento entre nombres singulares y plurales, los nombres singulares muestran un alineamiento nominativo-acusativo no prototípico, puesto que todos los argumentos dependientes se marcan con el mismo morfema $-t a^{l}$ : objeto directo (1b), objeto indirecto y sujeto de clausula subordinada si está es un objeto oracional. En cambio, los nombres plurales presentan un alineamiento neutro, debido a que todos los argumentos se marcan con el morfema de numero plural $-m$ (1c) y el morfema -ta desaparece en contextos de pluralidad (Estrada et al., 2013, Martínez, 2015).
a. inepo apo'ik bicha-k
1SG.NOM 2sG.ACU ver-PFV
«yo te vi» (Martínez, 2015).
b. tuka-ne Antonio-ta bicha-k
Ayer-1SG.NOM Antonio-DEP.SG ver-PFV
«ayer yo vi a Antonio» (Martínez, 2015).
c. yme jamuchi-m bicha-k
DET.PL hombre-PL mujer-PL ver-PFV
«los hombres vieron a las mujeres» (Martínez, 2015).

A continuación, se presenta el marco teórico y conceptual sobre los eventos de movimiento y los patrones lexicales $(\$ 2)$, posteriormente se muestra el análisis de los datos dividido en cinco apartados: los patrones lexicales de los eventos de movimiento en yaqui (§3), patrón lexical: movimiento y trayectoria (§4), patrón lexical: movimiento y manera $(\$ 5)$ patrón lexical: movimiento, trayectoria y causa $(\S 6)$, patrón lexical: movimiento, trayectoria y figura $(\S 7)$ y finalmente se exponen las conclusiones.

\footnotetext{
1. El sufijo - ta en yaqui es u morfema sincrético que además de ocurrir en el objeto de una construcción transitiva, el objeto indirecto de una construcción bitransitiva, el sujeto de una cláusula subordinada también ocurre en el elemento dependiente de una frase posesiva y una frase posposicional, es decir, el poseedor y el objeto de posposición respectivamente (Martínez 2015).
} 


\section{Los eventos de movimiento y los patrones lexicales}

De acuerdo con Talmy (1985, p. 61; 2007, p. 70), «un evento de movimiento es una situación en donde un objeto se mueve o es localizado respecto otro objeto». El esquema de un evento de movimiento se compone de dos estructuras: la estructura conceptual y la estructura superficial. La estructura conceptual corresponde a los componentes semánticos obligatorios y opcionales que constituyen el evento de movimiento. Los componentes semánticos obligatorios son figura, fondo, movimiento y trayectoria puesto que estos constituyen el evento más básico de movimiento. En cambio, los componentes semánticos opcionales son manera y causa porque estos añaden un evento causativo o de manera al evento de movimiento existente, formando así un evento complejo también llamado co-evento (Talmy 1985, 2007).

Componentes semánticos obligatorios de un evento de movimiento:

i) Movimiento: naturaleza dinámica o estática.

ii) Trayectoria: camino recorrido por la figura.

iii) Figura: entidad que se mueve.

iv) Fondo: entidad de referencia respecto a la cual se ubica la figura.

-Componentes semánticos opcionales de un evento de movimiento (coevento):

v) Manera: forma en que se realiza el movimiento.

vi) Causa: movimiento motivado por una entidad diferente a la figura.

Por otra parte, la estructura superficial de un evento de movimiento se conforma por las unidades gramaticales que constituyen la oración sintáctica: nombre, verbo, adposición, morfema, cláusula subordinada, etc. Cada una de las unidades gramaticales de la oración representa por lo menos a uno de los componentes semánticos del evento (véase el ejemplo 2).

\begin{tabular}{|c|c|c|c|c|c|}
\hline $\begin{array}{l}\text { Sara } \\
\text { nombre } \\
\text { figura }\end{array}$ & $\begin{array}{l}\text { caminó } \\
\text { verbo } \\
\text { movimiento }\end{array}$ & $\begin{array}{l}\text { hacia } \\
\text { preposición } \\
\text { trayectoria }\end{array}$ & $\begin{array}{l}\text { la casa } \\
\text { nombre } \\
\text { fondo }\end{array}$ & $\rightarrow$ & $\begin{array}{l}\text { estructura superficial } \\
\text { estructura conceptual }\end{array}$ \\
\hline
\end{tabular}

Sin embargo, tal como se aprecia en el ejemplo anterior, la correspondencia entre los componentes semánticos de un evento de movimiento y las unidades gramaticales no siempre tienen una relación biunívoca, puesto que: i) en un mismo evento de movimiento un componente semántico puede estar representado por diversas unidades gramaticales o, por lo contrario, ii) una sola unidad gramatical puede expresar la combinación o fusión de diversos componentes semánticos, como el verbo caminó en (1), el cual fusiona el componente semántico movimiento y manera. En este trabajo, nos centramos en el último punto, las fusiones semánticas verbales, es decir, los patrones lexicales.

Para Talmy (2007) un patrón lexical es la fusión de componentes semánticos que denota la raíz de un verbo 
Los patrones lexicales verbales en los eventos de movimiento en lengua yaqui

de movimiento, sin considerar ningún tipo de morfología derivativa que complemente su significado. De acuerdo con Talmy (1985) los patrones lexicales de movimiento más comunes en las lenguas del mundo son i) movimiento y trayectoria (coreano, turco, lenguas semíticas, lenguas romances); ii) movimiento y manera (chino, walpiri y lenguas indoeuropeas - excepto las romances -) y iii) movimiento y figura (navajo, atsugewi).

Autores como Talmy (1985, 2007), Levin (1993), Ibarretxe (2009) e Imbert (2012) refieren que una misma lengua tiene la capacidad de mostrar más de un patrón lexical, pues estos denotan los tipos verbales semánticos existentes en ella y cada uno de estos refiere a un esquema específico de movimiento.

En concreto, Imbert (2012) indica que los eventos de movimiento del inglés muestran al menos cuatro patrones lexicales verbales: i) movimiento y manera: verbos que denotan la forma en que una figura realiza el movimiento, p. ej., strode «entrar con pasos largos»; ii) movimiento y causa: verbos que refieren un movimiento provocado por una entidad diferente a la figura, p. ej., put «poner»; iii) movimiento, causa, fondo: verbos que expresan el fondo hacia donde se mueve la figura, p. ej., box «encajonar»; y iv) movimiento y figura: verbos que en su contenido semántico manifiestan la figura que se mueve, p. ej., rain «llover».

\section{Los patrones lexicales en los eventos de movimiento en yaqui}

En los eventos de movimiento de la lengua yaqui se distinguen cuatro patrones lexicales: i) movimiento y trayectoria; ii) movimiento y manera; iii) movimiento, trayectoria y causa; y iv) movimiento, trayectoria y figura. Los verbos que denotan el patrón lexical movimiento y trayectoria precisan el recorrido realizado por la figura, el cual puede ser hacia o desde el centro deíctico, como siika «ir, singular, pasado» en (3a). Los verbos que lexicalizan los componentes movimiento y manera indican la forma en que la figura realiza el movimiento, como buite «correr» en (3b). Los verbos que expresan en la raíz verbal el componente movimiento, trayectoria y causa manifiestan que el movimiento de la figura es provocado por una entidad distinta a esta, como toja «llevar» en (3c). Finalmente, los verbos que muestran el patrón lexical movimiento, trayectoria y figura, tal como su nombre lo indica, exponen en su contenido semántico la figura y el camino recorrido por esta, como ba'ata «acarrear agua» en (3d). Entiéndase que, en este último grupo verbal, la figura no se explicita de manera nominal en el evento.
a. Joan
Wa'imam-meu
siika
Juan Guaymas-DIR.PL ir.SG.PFV
«Juan fue a Guaymas» (Trabajo de campo, 2018).

$\begin{array}{llll}\text { b. } \begin{array}{l}\text { empo } \\ \text { 2SG.NOM } \\ \text { «tú corres al río» } \\ \text { río-DIR (Trabajo de campo, 2019). }\end{array} & \begin{array}{l}\text { buite } \\ \text { nadar }\end{array} \\ \text { c. } & \text { inepo } & \text { Bikam-meu } & \text { am }\end{array}$ toja-k 
Los patrones lexicales verbales en los eventos de movimiento en lengua yaqui

1SG.NOM Vícam-DIR.PL 2PL.ACU llevar-PFV

«yo los llevé a Vícam» (Trabajo de campo, 2018).

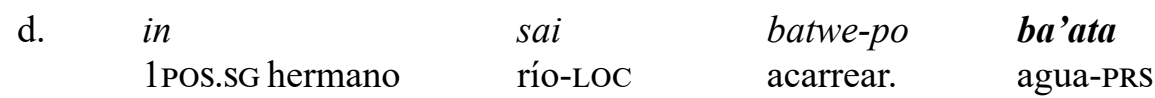

«mi hermano acarrea agua del río» (Diccionario yaqui-español, 2004).

En yaqui los eventos de movimiento se expresan en estructuras intransitivas o transitivas. Las estructuras intransitivas corresponden a las que lexicalizan en la raíz verbal movimiento y trayectoria, movimiento y manera o movimiento, trayectoria y figura. Este tipo de eventos se constituyen por el sujeto, el cual representa a la figura, el adjunto locativo, el cual representa al fondo, y por el verbo (4).

$\begin{array}{llcr}\text { in } \quad \text { ae } & \text { pajkoa- } \boldsymbol{u} & \text { siika } \\ \text { 1SG madre } & \text { fiesta-DIR } & \text { ir.PFV } \\ \text { «mi madre fue a la fiesta» (Trabajo de campo, 2018). }\end{array}$

Por su parte, las estructuras transitivas son las que fusionan en la raíz verbal el movimiento, trayectoria y la causa. Dichas estructuras se constituyen por el sujeto, el cual expresa la entidad causante del movimiento: el objeto directo, el cual representa a la figura, y al adjunto locativo, el cual a su vez denota al fondo, como en el verbo (5).

\begin{tabular}{|c|c|c|c|c|}
\hline & $\begin{array}{l}\text { maala } \\
\text { mamá }\end{array}$ & $\begin{array}{l}\text { lominko- } t \\
\text { domingo-LOC }\end{array}$ & $\begin{array}{l}\text { altaare- } \boldsymbol{u} \\
\text { altarer-DIR }\end{array}$ & $\begin{array}{l}\text { seewa-m } \\
\text { flor-PL }\end{array}$ \\
\hline
\end{tabular}

Tal como hemos visto en los ejemplos anteriores, los fondos de los eventos de movimiento en yaqui se expresan por medio de frases posposicionales, las cuales, como ya mencionamos, cumplen la función de un adjunto locativo. Las frases posposicionales se constituyen de una frase nominal sufijada por las posposiciones: $-u /$-wi «a/hacia» (4-5), -po «en/entre» (6a) o - $t$ «sobre» (6b).
a. ne
ousi juya-po
weama-n-pe'ea- $n$
1sG.NOM mucho monte-LOC
caminar-N-DES-PAS.IMPF
«me gustaba mucho andar en el monte» (Jeka Aniata Jiapsi, 2018).
b. tukaa-po te bo'o-t kateka chu'u-ta bicha-k
Noche-LOC 1PL camino-LOC caminar.PL-SUB Perro-DEP.SG ver-PFV
«en la noche, nosotros vimos un perro caminando sobre el camino» (Narraciones yaquis, 2002).

Dichas partículas $-u /-w i$ «a», -po «en» $\mathrm{y}-t$ «sobre» corresponden a las llamadas posposiciones ligadas de la lengua (Estrada, 2009; Guerrero, 2014). Por su parte, $-u$ introduce locaciones direccionales, mientras que 
Los patrones lexicales verbales en los eventos de movimiento en lengua yaqui

-po y - $t$ locaciones estáticas. La presencia de dichas partículas es obligatoria en la expresión de los eventos de movimiento, ya que establecen la relación semántica entre el verbo y el fondo (Guerrero, 2014; Martínez, 2021). Sin embargo, dichas posposiciones tienen significados canónicos, los cuales no siempre se conservan cuando ocurren en eventos de movimiento, puesto que estos se modifican dependiendo del contexto verbal en el que ocurran (Martínez, 2021).

A continuación, se describirá cada uno de los patrones lexicales presentes en la lengua yaqui, así como la estructura sintáctica y semántica de los eventos en que ocurre cada patrón.

\section{Patrón lexical i): movimiento y trayectoria}

El primer tipo verbal identificado en los eventos de movimiento, y el más recurrente, es el que fusiona en la raíz el componente semántico movimiento y trayectoria. El significado base de estos verbos denota el recorrido realizado por la figura para llegar a un fondo. Esto es, este tipo de eventos expresan el cambio de locación de la figura, tal como se aprecia en (7) donde la figura ne «yo» se dirige hacia el fondo telesecundariau «a la telesecundaria».

$\begin{array}{lllll}\text { (7) junak } & \text { into } & \text { ne } & \text { telesecundaria-u } & \text { kibake-k } \\ \text { entonces } & \text { CONJ } & \text { 1SG.NOM } & \text { telesecundaria-DIR } & \text { entrar-PFV } \\ & \text { «y entonces entré a la telesecundaria» (Jeka Aniata Jiapsi, 2018). }\end{array}$

En (8) se muestra una lista de los verbos más comunes que lexicalizan movimiento y trayectoria, en donde podemos apreciar que el grupo cuenta con formas verbales supletivas que distinguen entre el número gramatical, como entrar, caer y llegar, y entre el número gramatical y el tiempo, como el verbo ir (Dedrick \& Casad, 1999; Estrada, 2004; Guerrero, 2014).

$\begin{array}{ll}\text { Verbos de trayectoria más frecuentes } \\ \text { chitojte } & \text { «resbalar» } \\ \text { wo'okte } & \text { «hundirse» } \\ \text { wo'ote } & \text { «tirarse» } \\ \text { ja'amu } & \text { «subir» } \\ \text { jajase } & \text { «perseguir» } \\ \text { kibake } & \text { «entrar, singular» } \\ \text { kiimu } & \text { "entrar, plural» } \\ \text { kuusaroa } & \text { «cruzar» } \\ \text { noite } & \text { «acudir» } \\ \text { notte } & \text { «regresarse» } \\ \text { ropte } & \text { «zambullirse» } \\ \text { rukte } & \text { «moverse, aproximarse» } \\ \text { sajak } & \text { «ir, sujeto plural, pasado» } \\ \text { saka } & \text { «ir, sujeto plural, no pasado» }\end{array}$


Los patrones lexicales verbales en los eventos de movimiento en lengua yaqui

$\begin{array}{ll}\text { siika } & \text { «ir, sujeto singular, pasado» } \\ \text { siime } & \text { «ir, sujeto singular, no pasado» } \\ \text { so'ite } & \text { «alzarse» } \\ \text { watakte } & \text { «marcharse» } \\ \text { watte } & \text { "caer, plural» } \\ \text { weche } & \text { «caer, singular» } \\ \text { yaja / yaji } & \text { «llegar, sujeto plural» } \\ \text { yepsa } & \text { «llegar, sujeto singular» } \\ \text { yojte } & \text { «caerse (objetos)» }\end{array}$

Los eventos de movimiento que expresan verbos de este grupo muestran estructuras sintácticas intransitivas, constituidas por el sujeto (figura), el adjunto locativo (fondo) y el verbo. En estas estructuras la figura que se mueve siempre se corresponde con el sujeto de la oración, como ume yoemem «los hombres» y el fondo siempre se relaciona con el adjunto locativo, etriau «a la siembra» (9).

$\begin{array}{lccc}\text { ume } & \text { yoeme-m } & \text { etria-u } & \text { kiimu- } k \\ \text { DET.PL } & \text { hombre-PL } & \text { siembra-DIR } & \text { entrar.PL-PFV } \\ \text { «los hombres } & \text { entraron a la siembra» } & \text { (Trabajo de campo, } & \text { 2018). }\end{array}$

En este grupo verbal, los fondos ocurren sufijados por las posposiciones - $u$, -po o - $t$. La presencia de la posposición - $u$ en el fondo expresa la locación hacia donde se dirige la figura (10a). En cambio, la presencia de -po denota la locación hacia donde se adentra la figura (10b). Mientras que la ocurrencia del fondo con - $t$ indica que la figura se dirige a la cumbre de la locación (10c).
a. in ae pajkoa-u
yepsa-k
1SG madre fiesta-DIR llegar-PFV
«mi madre fue a la fiesta» (Trabajo de campo, 2018).
b. $u$ yoeme ba'a-po kibake-k
DET caballo agua-LOC entrar-PFV
«el entró en el agua» (Trabajo de campo, 2018).
c. $u$ chu'u kawi-t ja'amu
DET perro cerro-LOC subir.PRES
«el perro sube la montaña» (Trabajo de campo, 2018).


Cabe aclarar que la semántica de -po en (10b) no es la prototípica en la lengua, puesto que dicho sufijo canónicamente expresa locación estacionaria ${ }^{2}$ o locación temporal ${ }^{3}$. Sin embargo, su ocurrencia como locación direccional es común en eventos con verbos que lexicalizan la trayectoria y tienen un fondo al que la figura se adentra, como kibake «entrar, singular» o wo'okte «hundirse».

Una diferencia entre - $p o$ y las posposiciones $-u$ y $-t$ es que estas últimas concuerdan en número con el nombre de la frase posposicional. Esto es, si el fondo hace referencia a una locación plural, el nominal ocurrirá sufijado con el morfema plural - $m$. Asimismo, la posposición ligada mostrará concordancia de número prefijando el morfema -me «plural». —Véanse kawimmet «a las montañas» en (11a) y Potammeu «a Pótam (topos)» en (11b)—. La posposición -po nunca ocurre con locaciones plurales ni se pluraliza.
$1 \mathrm{SG}$ allá hacia RDP ir-PSD.IMPF
allá cerro-PL-LOC.PL
a. ne nam bichaa si $\sim$ siime-n
aman kawi-m-met
ja'amu-bae-kai
«me iba hacia allá a subir las montañas, allá» (Jeka Aniata Jiapsi, 2018).
b. bempo Pota-m-meu maka sako
3PL.NOM topo-PL-DIR.PL ir.pasado mañana
«ellos van a ir a Pótam ${ }^{4}$ pasado mañana» (Diccionario yaqui-español, 2004).

Finalmente, en algunos contextos de ocurrencia, los eventos que lexicalizan en la raíz verbal movimiento y trayectoria pueden ocurrir en estructuras que no explicitan el fondo, como siika «ir, singular, perfectivo» en (12a) o weche «caer» (12b). De acuerdo con Levin (1993), las oraciones con verbos de trayectoria tienden a expresar la locación hacia donde se realiza el movimiento, aunque en algunos contextos puede omitirse. Por ello, para comprobar que el verbo expresa de manera léxica del significado movimiento-trayectoria, basta con corroborar que el evento sigue implicando el desplazamiento de la figura, aunque la locación no se exprese. Tal como ocurre en yaqui, véanse los ejemplos en (11).

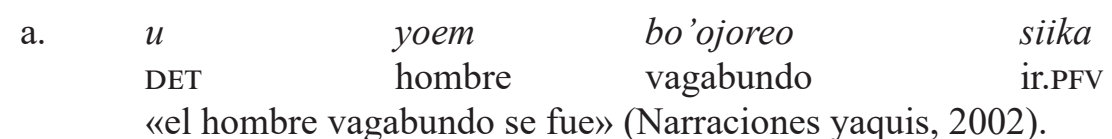

«el hombre vagabundo se fue» (Narraciones yaquis, 2002).

\begin{tabular}{|c|c|c|c|c|}
\hline 2. & $\begin{array}{l}\text { Juan } \\
\text { Juan } \\
\text { «Juan jue }\end{array}$ & $\begin{array}{l}\text { kora-po } \\
\text { corral-loc } \\
\text { lega en el corral». }\end{array}$ & $\begin{array}{l}\text { weeye } \\
\text { jugar }\end{array}$ & \\
\hline 3. & $\begin{array}{l}\text { In } \\
\text { 1sg.pos } \\
\text { «mi man }\end{array}$ & $\begin{array}{r}\text { maala } \\
\text { mamá } \\
\text { ná nació en } 1951\end{array}$ & $\begin{array}{l}\text { 1951-ро уеu } \\
\text { 1951-locafuera }\end{array}$ & $\begin{array}{l}\text { tomte-k } \\
\text { nacer-pfv }\end{array}$ \\
\hline
\end{tabular}

4. En la actualidad Pótam (topos) no se traduce al español, debido a que es el nombre propio de una localidad. 
$\begin{array}{lll}\text { b. } & \text { kora } & \text { weche-k } \\ \text { DET corral caer-PFV } & \text { «el corral se cayó» (Diccionario yaqui-español, 2004). }\end{array}$

\section{Patrón lexical ii): movimiento y manera}

El segundo patrón lexical que ocurre en los eventos de movimiento del yaqui corresponde al que fusiona en la raíz verbal el componente semántico movimiento y manera. Los verbos de este grupo se refieren a acciones que ponen énfasis en la forma en que se realiza la acción, es decir, en el patrón de movimientos realizados por la figura al moverse. Obsérvese el ejemplo en (13), donde ocurre el verbo aane «andar a pasos, singular».

$\begin{array}{llll}t a & \text { ne } & \text { kawi-u si } & \text { aane-pe'ea-n } \\ \text { pero } & \text { 1SG.NOM } & \text { cerro-DIR } & \text { INT andar.a.pasos-DES-PAS.IMPF } \\ \text { "pero me gustaba ir (andar) a la sierra» } & \text { (Jeka Aniata Jiapsi, 2018). }\end{array}$

En la lista de (14) se muestran los verbos de movimiento y manera más comunes en la lengua, en donde se aprecia que algunos verbos tienen formas supletivas que distinguen entre el número gramatical: andar, correr y caminar (Dedrick \& Casad, 1999; Estrada, 2004; Guerrero, 2014).

(14) Verbos de manera usados frecuentemente en lengua yaqui

$\begin{array}{ll}\text { aane } & \text { "andar a pasos, singular» } \\ \text { bajume } & \text { «nadar» } \\ \text { biakte } & \text { «rodarse» } \\ \text { bo'ojoa } & \text { «caminar (lit. hacer camino)» } \\ \text { buite } & \text { «correr, singular» } \\ \text { bwelta } & \text { "girar» } \\ \text { chepte } & \text { «brincarse» } \\ \text { chepta } & \text { «brincar» } \\ \text { jajasen } & \text { «perseguir» } \\ \text { kaate } & \text { «caminar, plural» } \\ \text { masaba'ite } & \text { «aletear» } \\ \text { ne'e } & \text { "Volar» } \\ \text { polakte } & \text { "trotar» } \\ \text { rejte } & \text { «andar a pasos, plural» } \\ \text { ro'akte } & \text { «revolcarse» } \\ \text { tenne } & \text { "correr, plural» } \\ \text { weye } & \text { «caminar, singular» } \\ \text { yooa } & \text { «temblar» }\end{array}$

Los eventos de movimiento que muestran verbos como los de (14) tienen una estructura sintáctica intransitiva, es decir, se forman de un sujeto (figura), un adjunto locativo (fondo) y un verbo. En estas estructuras, el fondo 
Los patrones lexicales verbales en los eventos de movimiento en lengua yaqui

exclusivamente ocurre sufijado con $-u$, puesto que dicha partícula es la única que intrínsecamente puede introducir el significado de trayectoria (15a), el cual es obligatorio en la expresión de eventos de movimiento. Por lo contrario, si el fondo ocurre sufijado con -po (15b) o - t (15c) el evento denotado será de locación y no de movimiento debido a que no hay trayectoria explícita en ningún elemento gramatical del evento.

(15) a
a. $\quad$ bea
juya-u
we-weama-n-ne
entonces 1SG.NOM monte-DIR RDP-caminar-PAS.IMPF-1SG.NOM
«entonces, yo iba (caminaba) al monte» (Jeka Aniata Jiapsi, 2018).
b. goi chu'u-m juya-po kaate
DET perro-PL monte-LOC caminar.PL.PRS
«dos perros van caminando en el monte» (Trabajo de campo, 2018).
c. juna'a suáwaka am ji-ji-ba-oteeka-t bwiti-ne
DEM.SG estrella.fugaz 3PL.ACU RED-alumbrar-DES-COND cielo-LOC correr-FUT
«cuando aquella estrella fugaz los quiera alumbrar, correrá sobre el cielo» (Narraciones yaquis, 2002).

Recordemos que el significado canónico de las posposiciones -po y - $t$ en yaqui es locativo, por tanto, en eventos con verbos de movimiento y manera, los fondos que ocurren sufijados con - $p o$ expresan una locación física en la que se mueve la figura (16a) y los fondos que ocurren sufijados con - $t$ manifiestan una locación sobre la que se mueve la figura (16b). Contrariamente, tal como se mostró en (§3), en eventos con verbos de movimiento y trayectoria estas partículas no indican fondos locativos sino tipos muy específicos de fondos direccionales.
a. empo batwe-po bajum
2SG rio-LOC nadar
«tú nadas en el río» (Trabajo de campo, 2018).

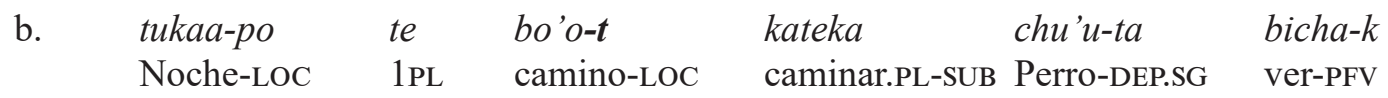

«en la noche, caminando sobre el camino, vimos a un perro» (Narraciones yaquis, 2002).

Por último, los verbos con patrón movimiento y manera pueden ocurrir en estructuras que no exhiben el fondo, tal como se aprecia en (17a) y (17b). Además, también pueden expresarse en estructuras transitivas (sujeto, objeto directo, verbo), tal como se observa en (17c) y (17d). En todos los casos anteriores, la figura siempre se corresponde con el sujeto; sin embargo, cabe destacar que estas estructuras tampoco se refieren a eventos de movimiento, sino a eventos locativos.
$\begin{array}{ll}\text { a. } & \text { empo } \\ & \text { 2SG.NOM }\end{array}$
$k a a$
bajume
nadar
«tú no nadas» (Diccionario yaqui-español, 2004). 
Los patrones lexicales verbales en los eventos de movimiento en lengua yaqui
b. empo
eтpo
ju'unea
tu' $i-s i$
o kaa tu'isi
weye-tek-o
2sg.NOM 2sg.NOM saber. PRS bien-MOD
«tú sabes si vas bien o no vas bien» (Jeka Aniata Jiapsi, 2018).
$\begin{array}{lllll}\text { c. } & u & \text { bake'o kaba'i-ta kawi-po jajase } \\ \text { DET vaquerocaballo-DEP.SG } & \text { sierra-LOC perseguir.PRS }\end{array}$
«el vaquero corretea al caballo en la sierra» (Trabajo de campo, 2018).
d. wakas kora-ta chepta
Vacas corral-DEP.SG brincar.PRS
«las vacas brincaron el corral» (Diccionario yaqui-español, 2004).

\section{Patrón lexical iii): movimiento, trayectoria y causa}

El tercer patrón verbal identificado en los eventos de movimiento en yaqui es el que tiene como base la fusión semántica movimiento, trayectoria y causa. Dichas raíces verbales denotan una situación en donde una entidad desplaza o mueve a la figura. Esto es, el movimiento de la figura no es volitivo sino provocado por otra entidad.

Las estructuras de los eventos de movimiento con verbos de movimiento causado son transitivas y se conforman por el sujeto, el objeto directo (figura), el adjunto locativo (fondo) y el verbo. En este tipo de eventos, a diferencia de los mostrados en $(\S 3)$ y ( $(4)$, la figura se relaciona con el objeto directo (18). Además, el adjunto locativo puede posicionarse antes del objeto, como altareu «al/hacia el altar» en (18a) o después del objeto, como Juantau «a/ hacia Juan» (18b).
a. in maala
lominko-t
altaare- $\boldsymbol{u}$
seewa- $m$
toji-bae
1POS.SG mamá
domingo-LOC altarer-DIR flor-PL
llevar-DES
«mi mamá va a llevar flores al altar» (Diccionario yaqui-español, 2004).
b.

$\begin{array}{lllll}u & \text { yoeme } & \text { kutam } & \text { Joan-ta- } \boldsymbol{u} & \text { rupta } \\ \text { DET } & \text { hombre } & \text { palo-PL } & \text { Juan-DEP.SG-DIR } & \text { acercar }\end{array}$

«el hombre acerca los palos a Juan» (Trabajo de campo, 2018).

En (19) se muestran los verbos de movimiento causado más comunes en la lengua. Cabe mencionar que este grupo verbal, al igual que los grupos verbales anteriores, tiene formas supletivas que distinguen entre el número gramatical, tal como lo muestran extraer y bajar.

(19) Verbos de movimiento causado de trayectoria más frecuentes

baau «extraer, objeto, singular»

go'okta «hundir»

jimaa «lanzar/aventar»

jittoja «acarrear»

kibacha «meter, objeto singular»

kiima «meter, objeto plural» 
Los patrones lexicales verbales en los eventos de movimiento en lengua yaqui

$\begin{array}{ll}\text { koota } & \text { «extraer, objeto plural»* } \\ \text { naama } & \text { «arrear» } \\ \text { nu'upa } & \text { «traer» } \\ \text { rukta } & \text { «acercar» } \\ \text { so'ita } & \text { «alzar» } \\ \text { suulu } & \text { «deslizar» } \\ \text { suume } & \text { «bajar un líquido» } \\ \text { tatabek } & \text { «tumbar» } \\ \text { tojak } & \text { «llevar» } \\ \text { watta } & \text { «arrojar» } \\ \text { weiya } & \text { «llevar» } \\ \text { wike } & \text { «jalar» } \\ \text { yecha } & \text { «poner» } \\ \text { yojta } & \text { «bajar» }\end{array}$

Una característica de los eventos de movimiento que expresan este tipo de verbos es que la concordancia de número de las formas supletivas no se corresponde con el sujeto, como en los verbos de trayectoria (\$3) y los verbos de manera (§4), sino con el objeto directo, el cual representa a la figura. En (20a) vemos el verbo kibacha «meter, singular» concordando en número con el objeto nee «a mí». En contraste, en (20b) apreciamos la forma verbal kiima «meter, plural» concordando con el objeto directo wakasim «vacas».
a. nee
ejkuela-u
kibacha-wa-ka=nee
1SG.ACU escuela-DIR
meter.SG-IMPR-PFV=1SG.ACU
«me metieron a la escuela» (Jeka Aniata Jiapsi, 2018).
b. Joan wakasi-m kora-u kiima-k
Juan vaca-PL corral-DIR meter.PL-PFV

«Juan metió las vacas al corral» (Trabajo de campo, 2018).

Respecto a los fondos de estos eventos, se comportan de la misma forma que los fondos de los eventos con verbos de movimiento y trayectoria. Esto es, se sufijan con la posposición direccional -u/-meu cuando el fondo denota la locación hacia donde es movida la figura (21a) o con -po cuando el fondo refiere a la locación hacia donde la figura es introducida, como en (21b).
a. inepo Bikam-meu am tojak
1SG Vícam-DIR.PL 3PL llevar-PFV
«yo los llevé a Vícam» (Diccionario yaqui-español, 2004).
b. in sai karo-ta techoa-po go'okta
«mi hermano hunde el carro en el lodo» (Trabajo de campo, 2018).
1SG.POS hermano carro-DEP.SG lodo-LOC hundir-PRES

Del mismo modo, al igual que en los eventos con verbos de trayectoria, algunas estructuras de movimiento causado pueden no expresar el fondo por ser recuperable del contexto, pero el sentido de trayectoria sigue presente 
en los verbos (22).

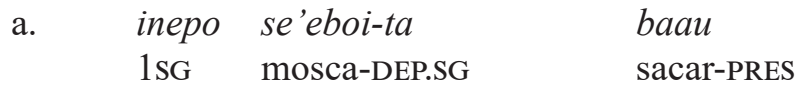
«estoy sacando la mosca» (Trabajo de campo, 2018).

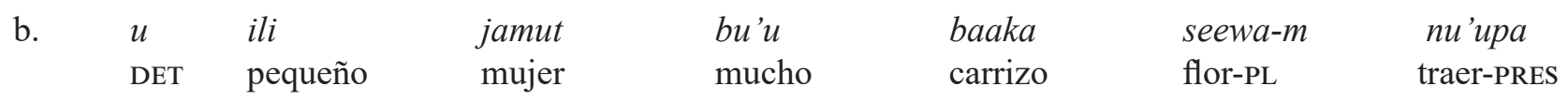
«la muchacha trae muchas flores de carrizo» (Diccionario yaqui-español, 2004).

Por otra parte, una particularidad de los fondos de los eventos de movimiento con verbos causativos es que diferencian entre entidades inanimadas y entidades animadas. Por tanto, si el fondo corresponde a una locación direccional inanimada, el nominal se acompaña con la partícula -u/meu «a/hacia», tal como vemos en (23a). No obstante, si el nominal corresponde a una entidad animada, este prefija el morfema $-t a$ al direccional $-u$, como podemos observar en (23b). En este punto, cabe resaltar que en la introducción de este escrito, se mencionó que el sufijo - ta en la lengua yaqui es un morfema sincrético ${ }^{5}$, cuyo común denominador es que siempre ocurre con nominales de valor argumental, sintácticamente dependientes y singulares(Martínez, 2015). Por tanto, consideramos que la ocurrencia de - $t a$ en contextos como el de (23b) denota que un fondo animado es jerárquicamente más relevante que el inanimado, por lo cual se considera más argumental que un adjunto prototípico sin -ta.

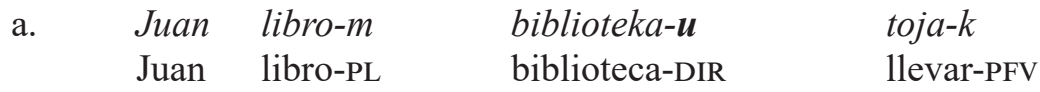
«Juan llevó los libros a biblioteca» (Martínez, 2015).
b. $u$ unsi jamut jiosiam maejtro-ta-u nu'upa-k
DET pequeño niño mujer libro maestra-DEP.SG-DIR traer-PFV «la niña trajo el libro a la maestra» (Martínez, 2015).

Entre los verbos que lexicalizan el movimiento y la causa es posible encontrar formas que tienen como tercer componente semántico la manera, como biakta «rodar» y ro’akta «revolcar». No obstante, en eventos con este tipo de verbos solamente pudimos identificar estructuras en donde el fondo ocurría sufijado con -po «en» denotando eventos de locación y no de movimiento (24), del mismo modo que en los eventos con verbos de manera (§4).

$\begin{array}{lccc}\text { inepo } & \text { teta-ta } & \text { bwia-po } & \text { biakta } \\ \text { 1SG.NOM } & \text { piedra-DEP.SG } & \text { tierra-LOC } & \text { rodar-PRS } \\ \text { «yo estoy rodando la piedra en la tierra» } & \text { (Trabajo de campo, 2018). }\end{array}$

Asimismo, en este tipo de eventos de movimiento causado identificamos tres formas verbales distintas que refieren tres maneras de expresar el verbo «llevar»: iba'ate «llevar abrazado», ke'ebete «llevar mordido»y

5. El sufijo - $t a$ puede ocurrir con el objeto directo, el objeto indirecto, el complemento de algunas posposiciones, el poseedor de la frase posesiva o con el sujeto de las cláusulas subordinadas (Martínez, 2015). 
Los patrones lexicales verbales en los eventos de movimiento en lengua yaqui

machu'ute «llevar en las manos». Estas formas verbales ocurren en estructuras sintácticas transitivas, a pesar de que tienen la terminación que exhiben los verbos intransitivos ${ }^{6}$, la cual es -te. Respecto a la figura, esta se sigue correspondiendo al objeto directo (25), al igual que en todos los eventos con movimiento causado. Es importante resaltar, pues, que en estos ejemplos los fondos nunca estuvieron explícitos.
a.
$\begin{array}{ll}u & \text { yoeme } \\ \text { DET } & \text { hombre } \\ \text { «el hombre lleva } & \\ u & \text { jamut }\end{array}$
jamu-ta
iba'ate
DET hombre mujer -DEP.SG llevar.abrazado-PRS
«el hombre lleva abrazada a la mujer» (Diccionario yaqui-español, 2004).
b. $u$ jamut kuta-ta machu'ute
llevar.en.las.manos-PRS
«la mujer lleva en las manos un palo» (Diccionario yaqui-español, 2004).

Las formas verbales en (25) tienen alternativas transitivas, las cuales terminan en -ta, al igual que la mayoría de los verbos transitivos de la lengua, pero estas no denotan movimiento. Véanse los ejemplos de (26).

(26) Contraste entre las formas intransitivas e transitivas

$\begin{array}{lllll}\text { Iba'ate } & \text { «llevar abrazado» } & \rightarrow & \text { ibakta } & \text { «abrazar» } \\ \text { Ke'ebete } & \text { «llevar mordido» } & \rightarrow & \text { ke'ebeta } & \text { «morder» }\end{array}$

\section{Patrón lexical iv): movimiento, trayectoria y figura}

El último patrón lexical de la lengua fusiona en el verbo el movimiento la trayectoria y la figura. Estas formas se caracterizan por no expresar gramaticalmente la figura, debido a que esta se manifiesta en la semántica verbal. En este grupo podemos encontrar generalmente verbos que provienen de nominales de elementos naturales, como el agua, la tierra o la lluvia, aunque también ocurren verbos relacionados con fenómenos naturales. Véanse los ejemplos de (27).

(27) Verbos más frecuentes de trayectoria y figura

$\begin{array}{lllll}\text { Ba'ata } & \text { «acarrear agua» } & \rightarrow & \text { baa'am } & \text { «agua» } \\ \text { Bwakta } & \text { «sacar tierra» } & \rightarrow & \text { bwia } & \text { «tierra» } \\ \text { Yuke } & \text { «llover» } & \rightarrow & y u k u & \text { «lluvia» } \\ \text { Be'okte } & \text { «relampaguear» } & \rightarrow & ----- & \\ \text { Siijo'ote } & \text { «loviznar» } & \rightarrow & ----- & \end{array}$

Los eventos de movimiento que muestran estas raíces léxicas exhiben dos tipos de estructuras. La primera corresponde a estructuras intransitivas con sujeto explícito, en donde la figura está significada a través del verbo

6. Al respecto, Tubino (2010) menciona que las formas verbales terminadas en -te corresponden a la valencia intransitiva, mientras que las terminadas en - $t a$ corresponden a la valencia transitiva. 
Los patrones lexicales verbales en los eventos de movimiento en lengua yaqui

junto con el movimiento y la trayectoria, mientras que el fondo se expresa en una frase posposicional, donde el nominal se sufija con la posposición direccional $-u$. Debido a que el sujeto representa a la entidad que realiza el movimiento, estas estructuras pueden considerarse también como un tipo de movimiento causado (28).

$$
\begin{array}{lcl}
\text { in } \quad \text { sai } & \text { batwe- } \boldsymbol{u} & \text { ba'ata } \\
\text { 1SG.POS hermano } & \text { rio-DIR } & \text { acarrear.agua } \\
\text { «mi hermano acarrea agua del río» (Diccionario yaqui-español, 2004). }
\end{array}
$$

La segunda estructura que involucra verbos de figura es la intransitiva-impersonal. Esto se da porque las raíces verbales en este grupo se refieren a fenómenos naturales, los cuales habitualmente no tienen un sujeto. Asimismo, en lugar de fondos, los eventos de este tipo prefieren mostrar complementos adverbiales de cantidad, tal como se puede apreciar en (29a) con ousi «mucho» o complementos temporales, como ian «hoy» en (29b).
$\begin{array}{lll}\text { a. } & \text { ousi } & \text { yuku- } k \\ & \text { mucho } & \text { llover-PFV }\end{array}$
«llovió mucho» (Diccionario yaqui-español, 2004).
b. ian siijo'ote
Hoy lloviznar
«hoy está lloviznando» (Diccionario yaqui-español, 2004).

Finalmente, hay que resaltar el caso del verbo be'okte «relampaguear», el cual ocurre en una estructura sintáctica diferente a la de los otros verbos de fenómenos naturales, puesto que esta sí muestra explícito el sujeto. Esto se da debido a que dicho verbo literalmente significa «sacar la lengua» $y$, por tanto, su estructura requiere de un sujeto causa que realice el movimiento de la figura lexicalizada en el verbo (30).

$$
\begin{aligned}
& u \quad \text { yuku ousi be'okte } \\
& \text { DET lluvia mucho sacar.la.lengua } \\
& \text { «está relampagueando mucho (la lluvia saca mucho la lengua)» (Diccionario yaqui-español, 2004). }
\end{aligned}
$$

\section{Conclusiones}

En los eventos de movimiento en lengua yaqui se distinguen principalmente cuatro patrones lexicales verbales: i) movimiento y trayectoria; ii) movimiento y manera; iii) movimiento, trayectoria y causa; y iv) movimiento, trayectoria y figura. La ocurrencia de un patrón lexical u otro en los eventos de movimiento tiene implicaciones sintácticas y semánticas

Por un lado, los eventos de movimiento con patrones lexicales i), ii) y iv) muestran una estructura intransitiva, en donde el sujeto de la oración se corresponde con la figura del evento. En cambio, los eventos con el patrón lexical iii) tienen una estructura transitiva, en donde el objeto directo de la oración se corresponde con la figura 
Los patrones lexicales verbales en los eventos de movimiento en lengua yaqui

del evento. Asimismo, detectamos que muchas de las unidades verbales poseen formas supletivas que distinguen entre número y tiempo. Es importarte recalcar que dichas formas supletivas siempre tienen concordancia con la figura del evento de movimiento.

Por otra parte, identificamos que los fondos de los eventos de movimiento se representan por adjuntos locativos, los cuales se constituyen por una frase nominal y una posposición ligada, que preferentemente tiende a ser $-u$, puesto que de manera canónica esta posposición es la única que introduce locaciones direccionales.

No obstante, en eventos de movimiento que muestran patrones lexicales i) o iii), los fondos también pueden ocurrir con las posposiciones canónicamente locativas - po y - $t$, denotando así locaciones direccionales, aunque esto solamente ocurre cuando las características verbales requieren de un fondo donde la figura pueda adentrarse.

Por último, cabe mencionar que las estructuras de movimiento en donde los patrones lexicales son ii) o iii) $\mathrm{y}$, además, los fondos ocurren marcados con las posposiciones -po o - $t$, no denotan eventos de movimiento, sino eventos de locación, puesto que en ningún elemento gramatical del evento se denota el significado de trayectoria. 


\section{Referencias bibliográficas}

1. Bejípone Cruz, M. (2013). Préstamos lingüisticos en la lengua yaqui. (Tesis de maestría). Hermosillo: Universidad de Sonora.

2. Dedrick, J. \& Casad, H. (1999). Sonora Yaqui Language Structures. Tucson: University of Arizona Press.

3. Estrada-Fernández, Z., Buitimea Valenzuela, C., Gurrola Camacho, A., Castillo Celaya, M. y Carlon Flores, A. (2004). Diccionario yaqui-español y textos. Obra de preservación lingüística. Hermosillo: Plaza y Valdéz/ Universidad de Sonora

4. Estrada-Fernández, Z., Carlón Flores, A. y Gutiérrez Estrada, M. R. (2018). Jeka Aniata jiapsi: Jiak jamutta etejoi. / Vida de Jeka Ania: Historia de una mujer yaqui. Hermosillo: Universidad de Sonora.

5. Estrada-Fernández, Z., Tubino Blanco, M., \& Villalpando Quiñonez, J. (2013). Yaqui valency patterns. Valency Patterns Leipzig. Leipzig: Max Planck Institute for Evolutionary Anthropology. www.valpal.info/languages/yaqui 6. Guerrero, L. (2014). Verbos de movimiento y predicados complejos en yaqui. En L. Guerrero (Coord.), Movimiento y espacio en lenguas de América (pp. 238-275). México: Universidad Nacional Autónoma de México. 7. Hernandez, G. (2002). Marcadores discursivos: una exploración en la narrativa yaqui (Tesis de Maestría). Hermosillo: Universidad de Sonora.

8. Ibarretxe-Antuñano, I. (2009). Path Salience in Motion Events. In J. Guo, E. Lieven, N. Budwig, S. Ervin-Tripp, K. Nakamura, \& S. Özçalişkan (Eds.), Crosslinguistic Approaches to the Psychology of Language: Research in the Tradition of Dan Isaac Slobin (pp. 403-414). New York: Psychology Press.

9. Imbert, C. (2012). Path: Ways Typology Has Walked Through It. Language and Linguistics Compass, 6(4), 236-258.

10. Lerma Rodríguez, E. (2014). Algunas consideraciones sobre investigación etnográfica en la tribu yaqui. Culturales, 2(2), 41-62.

11. Levin, B. (1993). English Verb Classes and Alternation. A Preliminary Investigation. Chicago: University of Chicago Press.

12. Lindenfeld, J. (1973). Yaqui Syntax. Los Angeles: University of California Press.

13. Martínez, D. (2015). El sufijo -ta en yaqui: redireccionando su clasificación (Tesis de Maestría). Hermosillo: Universidad de Sonora.

14. Martínez, D. (2021). Los eventos de movimiento en yaqui (Tesis de Doctorado). Hermosillo: Universidad de Sonora.

15. Moctezuma Zamarrón, J. (2007). Yaquis. Comisión Nacional para el Desarrollo de los Pueblos Indígenas. 16. Talmy, L. (1985). Lexicalization Patterns: Semantic Structure in Lexical Forms. Language Typology and Syntactic Description, 3(99), 36-149.

17. Tubino Blanco, M. (2010). Las causativas léxicas del yaqui. En Z. Estrada y A. Álvarez (Eds.), Estudios de lenguas amerindias (pp. 235-252). Hermosillo: Universidad de Sonora. 